\title{
Auditoria em saúde e economia da saúde: análise de um estudo de caso
}

Audit in health and health economics: analysis of a case study

Auditoría en salud y economía de la salud: análisis de un estudio de caso

\author{
André Luis Alves de Quevedo ${ }^{1}$ \\ Rodrigo Mendes Leal ${ }^{2}$
}

\begin{abstract}
Resumo
Objetivo: analisar os resultados da auditoria em saúde na área de órteses, próteses e materiais especiais (OPM) e cirurgias múltiplas e sequenciais (CMS), realizada pela Secretaria Estadual de Saúde do Rio Grande do Sul (SES/RS). Metodologia: estudo quantitativo descritivo, baseado em dados secundários da SES/RS, na área de OPM e CMS, referente ao período da prática de auditoria de pós-pagamento, de março de 2013 a janeiro de 2017, utilizando a abordagem estudo de caso. Resultados: foram analisadas 1.004 observações concluídas para o período; o número de processos administrativos com solicitação de devolução de recursos financeiros não teve uma tendência na série histórica analisada, resultado influenciado pela presença de processos administrativos não conclusos, em especial a partir de 2014, relativos à execução da auditoria ou até mesmo a judicialização do assunto pelas instituições auditadas. Quanto ao valor financeiro solicitado para devolução dos processos analisados, foi maior em 2014, decrescendo em 2015 e diminuiu consideravelmente nos anos 2016 e 2017. Considerando as 30 regiões de saúde do estado do Rio Grande do Sul, três instituições hospitalares tiveram destaque, a primeira com 43 processos administrativos com solicitação de devolução de recursos financeiros; a segunda com 30; e a terceira com 28. Conclusão: as ações em saúde, nessa área, podem ser qualificadas por meio de atenção especial para as regiões de saúde e instituições hospitalares que mais reincidem e tem grandes volumes financeiros glosados.
\end{abstract}

Palavras-chaves: Auditoria médica. Economia da saúde. Gestão em saúde. Judicialização da saúde. Sistema único de Saúde.

\begin{abstract}
Objective: To analyze the results of the health audit in the area of orthoses, prostheses and special materials (OPM), multiple and sequential surgeries (CMS), performed by the State Health Department of Rio Grande do Sul, Brazil (SES/RS). Methodology: Descriptive quantitative study, based on secondary data from SES/RS, in the area of OPM and CMS, referring to the period of post-payment audit practice, from March 2013 to January 2017, using the case study approach. Results: 1,004 completed observations were analyzed for the period; the number of administrative processes requiring a return of financial resources did not have a tendency in the analyzed historical series, a result influenced by the presence of administrative processes that were not conclusive, especially from 2014 , on the execution
\end{abstract}

\footnotetext{
${ }^{1}$ Mestrado em Epidemiologia pelo Programa de Pós-Graduação em Epidemiologia da Faculdade de Medicina da UFRGS; lotado na Secretaria Estadual de Saúde do Rio Grande do Sul. E-mail: andrequevedo_sls@hotmail.com

2 Doutor em Políticas Públicas, Estratégias e Desenvolvimento (PPED) pelo Instituto de Economia da UFRJ. Gerente na Área Social do BNDES. E-mail: rmendesleal@gmail.com
} 
of the audit or even the judicialisation of the subject audited institutions. As for the financial value requested for the return of the analyzed processes, it was higher in 2014, decreasing in 2015 and decreasing considerably in the years 2016 and 2017. Considering the 30 health regions of the state of Rio Grande do Sul, Brasil, three hospitals were highlighted, the first with 43 administrative processes requesting the return of financial resources; the second with 30; and the third with 28. Conclusion: health actions in this area can be qualified through special attention to the regions of health and hospital institutions that repeat more and have large financial volumes glossed.

Key-words: Medical audit. Health economics. Health management. Health's Judicialization. Unified Health System.

\section{Resumen}

Objetivo: Analizar los resultados de la auditoría de salud en el área de ortesis, prótesis y materiales especiales (OPM), cirugías múltiples y secuenciales (CMS), realizadas por el Departamento de Salud del Estado de Rio Grande do Sul, Brasil (SES / RS). Metodología: estudio cuantitativo descriptivo, basado en datos secundarios de SES / RS, en el área de OPM y CMS, en referencia al período de la práctica de auditoría posterior al pago, desde marzo de 2013 hasta enero de 2017, utilizando el enfoque de estudio de caso. Resultados: se analizaron 1,004 observaciones completas para el período; el número de procesos administrativos que requirieron un retorno de los recursos financieros no tuvo una tendencia en las series históricas analizadas, un resultado influenciado por la presencia de procesos administrativos que no fueron concluyentes, especialmente a partir de 2014, en la ejecución de la auditoría o incluso la judicialización del sujeto Instituciones auditadas. En cuanto al valor financiero solicitado para el retorno de los procesos analizados, fue mayor en 2014, disminuyó en 2015 y disminuyó considerablemente en los años 2016 y 2017. Considerando las 30 regiones de salud del estado de Rio Grande do Sul, Brasil, se destacaron tres hospitales, el primero con 43 procesos administrativos solicitando la devolución de recursos financieros; el segundo con 30; y el tercero con 28. Conclusión: las acciones de salud en esta área se pueden calificar a través de una atención especial a las regiones de salud y las instituciones hospitalarias que repiten más y tienen grandes volúmenes financieros.

Palabras-llave: Auditoría Médica. Economía de la Salud. Gestión en Salud. Judicialización de la Salud. Sistema Único de Salud.

\section{Introdução}

A auditoria em saúde é uma atividade que vem sofrendo transformações ao longo do período histórico. Conforme trazido pela Política Nacional de Gestão Estratégica e Participativa no SUS (ParticipaSUS), "a auditoria é um instrumento de gestão para fortalecer - Sistema Único de Saúde (SUS), contribuindo para a alocação e utilização adequada dos recursos, a garantia do acesso e a qualidade da atenção à saúde oferecida aos cidadãos" (1). 
Entre as atribuições da auditoria em saúde está a verificação dos processos e resultados referentes à prestação de serviços, considerando o modelo de atenção em saúde e as legislações vigentes em um determinado território (2).

De acordo com Costa (3), as reformas administrativas e financeiras que vêm acontecendo nos municípios em Portugal fazem com que a auditoria se torne um instrumento para qualificação da gestão pública, contribuindo para que se torne mais eficaz, eficiente e transparente.

Pensar de que forma a auditoria em saúde contribui para a organização do SUS pode ser uma indicação da eficiência e efetividade, bem como uma forma de melhor contribuir com a utilização dos recursos financeiros, buscando otimizar o sistema público de saúde.

Além disso, a auditoria pode servir como um instrumento de qualificação para o controle do serviço público de saúde prestado ao cidadão e gerar aprendizado para a gestão, a fim de subsidiar o aprimoramento da oferta de serviços de saúde, que em maior quantidade e qualidade tende a reduzir o número de usuários que não conseguem atendimento $\mathrm{e}$ precisam recorrer à judicialização

Na Lei n.․ 8.080, de 19 de setembro de 1990, artigo 16, foi prevista a criação do Sistema Nacional de Auditoria (SNA), que tem o papel, nas instâncias de gestão do SUS, de acompanhar, controlar e avaliar as ações e serviços de saúde (4).

O SNA configura-se como "o conjunto de órgãos e unidades instituídos em cada esfera de governo, sob a direção do gestor local do Sistema Único de Saúde, com atribuição de realizar auditorias" (5). No SNA, as fases de uma auditoria são as seguintes: fase analítica; fase operativa ou in loco; e fase do relatório final (6).

Pode-se considerar que "as ações de auditoria estão voltadas para o diagnóstico e a transparência, [...] possibilitando o acesso da sociedade às informações e resultados das ações do SNA e consolidando a auditoria como instrumento de gestão." (1).

No estado do Rio Grande do Sul (RS), a auditoria em saúde foi instituída pela Lei Estadual ํo 11.867, de 11 de dezembro de 2002 (7), que criou os procedimentos administrativos do Sistema Estadual de Auditoria e as medidas aplicáveis às irregularidades ocorridas no SUS do RS. Esse ato configurou-se como um marco normativo e parâmetro, pois foi o instrumento para a regularização da atividade no território do estado. 
A Coordenação de Auditoria Médica da Secretaria Estadual de Saúde do Rio Grande do Sul (CAME/SES/RS), em dezembro de 2017, estava organizada em auditoria médica, de enfermagem e contábil, com aproximadamente 70 auditores, sendo 60 médicos. As penalidades administrativas que os auditores, no exercício do seu trabalho, podem aplicar administrativamente - sem prejuízo das ações penais e cíveis - são: advertência; multa; devolução do valor pago a municípios ou a instituições prestadoras de serviço ao SUS/RS; suspensão do pagamento de qualquer forma de atendimento do SUS em caráter de internação e de ambulatório; suspensão temporária ou definitiva de repasse de recursos do Tesouro do Estado; e ainda suspensão do atendimento ou exclusão do credenciamento ao SUS no Estado (7).

A atuação da CAME/SES/RS se dá, principalmente, nas situações de óbito infantil; órteses, próteses e materiais especiais (OPM) e cirurgias múltiplas e sequenciais (CMS); sobreposto (mesma autorização de internação hospitalar é apresentada para mais de um pagamento); e tuberculose. Cabe enfatizar que, no caso de OPM e CMS, as auditorias são realizadas em todas as autorizações de internação hospitalar desse tipo e por auditores médicos.

Até o período de fevereiro de 2013 os procedimentos de OPM e CMS eram objeto de auditorias de pré-pagamento, ou seja, com as etapas de bloqueio, auditoria e pagamento ou outro procedimento cabível. Posteriormente, de fevereiro de 2013 e até janeiro de 2017, passaram a ser pagas e depois auditadas, ou seja, auditoria pós-pagamento. Essa mudança ocorreu para agilizar o pagamento e liberação dos recursos para os prestadores (instituições hospitalares). A partir de fevereiro de 2017 , cerca de $80 \%$ das coordenadoras regionais de saúde (CRS) voltaram a ter auditorias de pré-pagamento em, conforme deliberação superior da SES/RS.

No que se refere à área de órteses, próteses e materiais especiais e cirurgias múltiplas e sequenciais, existe um controle interno de dados administrativos da CAME/SES/RS sistematizado em planilha de Excel, desde o março de 2013 até janeiro de 2017 -, que,até o momento do início da presente pesquisa, tinha sido utilizado somente em análises internas para a operacionalização da auditoria.

Tendo-se em consideração que os recursos em saúde são escassos (8) e as necessidades em saúde, crescentes, a auditoria em saúde pode ser uma ferramenta para 
qualificação do SUS, especificamente no estado do Rio Grande do Sul. Considerando o exposto, o objetivo do presente artigo foi analisar os resultados da auditoria em saúde na área de órteses, próteses e materiais especiais, cirurgias múltiplas e sequenciais realizada pela Secretaria Estadual de Saúde do Rio Grande do Sul.

\section{Metodologia}

Foi desenvolvido um estudo quantitativo descritivo, baseado em dados secundários da Coordenação de Auditoria Médica da Secretaria Estadual de Saúde do Rio Grande do Sul, na área de órteses, próteses e materiais especiais (OPM) e cirurgias múltiplas e sequenciais (CMS), utilizando a abordagem de estudo de caso.

O estudo de caso é uma investigação empírica que investiga um fenômeno contemporâneo dentro de seu contexto real. Emprega-se, especialmente quando os limites entre o fenômeno e o contexto não estão claramente definidos. O estudo de caso costuma utilizar pelo menos duas técnicas de coleta de dados: a entrevista e a observação, mas pode também utilizar outras técnicas como, por exemplo, o questionário e a análise de documentos (9).

Foi utilizada para a análise uma planilha de Excel, empregada especificamente para o controle das auditorias em OPM e CMS, das instituições auditadas pela Coordenação de Auditoria Médica da Secretaria Estadual de Saúde do Rio Grande do Sul. Nessa planilha, constam 1.891 observações de processos administrativos de auditorias em saúde, com irregularidades/inconformidades identificadas em autorizações de internação hospitalar pagas (AIH), na área de OPM e CMS, referente ao período de março de 2013 a janeiro de 2017, ou seja, todos os dados disponíveis para o período de auditoria pós-pagamento.

As seguintes variáveis da base de dados foram utilizadas para este trabalho: data de registro do processo administrativo com ordem de recolhimento; prestador auditado; e valor solicitado para devolução (via ordem de recolhimento).

Para o levantamento dos dados do número de leitos (porte hospitalar), foi utilizado o Cadastro Nacional de Estabelecimentos de Saúde (CNES) (10); e para a categorização das informações de região de saúde das instituições hospitalares, foram consultadas as resoluções da Comissão Intergestores Bipartite (CIB/RS) n. ${ }^{\circ}$ 555, de 19 de setembro de 2012 (11), e n. ${ }^{\circ}$ 499, de 25 de setembro de 2014 (12). As tabelas, quadros e gráficos foram 
elaboradas nos aplicativos Excel e Word da Microsoft $\AA$; e os mapas, no Tabwin®/DATASUS/Ministério da Saúde/Brasil e Paint.

Conforme o Manual Sistema Débito Web, do Tribunal de Contas da União (TCU), Sistema de Atualização de Débito (13), até a data de 31/07/2011, os valores de débitos eram calculados com atualização monetária do Índice de Preços ao Consumidor Amplo (IPCA), acrescido dos juros simples de mora de $1 \%$ ao mês. A partir de $01 / 08 / 2011$, os débitos passaram a ser atualizados exclusivamente pela taxa SELIC (Sistema Especial de Liquidação e de Custódia).

Seguindo essa lógica, os dados dos processos administrativos com ordem de recolhimento de auditorias em saúde com irregularidades/inconformidades identificadas em AlH na área OPM e CMS pagas, da CAME/SES/RS, sofreram atualização monetária por meio da taxa SELIC, na data de seu registro na planilha, calculada via sistema de atualização de débito do TCU.

O projeto de pesquisa foi submetido ao Comitê de Ética e Pesquisa (CEP), da Escola de Saúde Pública do estado do Rio Grande do Sul, e aprovado, com parecer consubstanciado CEP número 2.451.950, em 21 de dezembro de 2017.

\section{Resultados}

Das 1.891 observações disponíveis na planilha, 1.004 puderam ser empregadas para análise. Não foram consideradas na análise 310 observações que, após o período de defesa, foram deferidas, ou seja, o devido pagamento foi considerado adequado; e 577 observações $(30,45 \%)$ por falta de completude das informações (perdas para o presente estudo), pelos processos estarem em andamento. Quanto a esses processos em andamento, em 2013 ocorreram 27 observações; em 2014, 118 observações; em 2015, 127 observações; em 2016, 213 observações; em 2017, 92 observações (Quadro 1).

As perdas mais expressivas de informações referentes aos anos de 2016 e 2017 se devem ao fato de que os valores financeiros dos processos administrativos ainda estão em período de devolução, ou, após o encerramento das etapas de auditoria, foram judicializados pelos prestadores de serviços.

Assim, as observações empregadas no aprofundamento da análise se referem aos processos concluídos para devolução, ou seja, aqueles em que foi emitida ordem de recolhimento 
(OR). Quanto a essas observações, no ano de 2013 foram identificadas 268, enquanto, por sua vez, 357 para 2014, 267 para 2015, 108 para 2016 e 4 para 2017 (Quadro 1).

Quadro 1. Quantidade (e percentual) de processos administrativos com notificação de irregularidade/inconformidade nas Autorizações de Internações Hospitalares de órteses, próteses e materiais especiais, cirurgias múltiplas e sequenciais pagas, realizados pela SES/RS, segundo estágio de auditoria, por ano

\begin{tabular}{|l|c|c|c|c|c|c|}
\hline \multicolumn{1}{|c|}{$\begin{array}{c}\text { Estágio da } \\
\text { Auditoria }\end{array}$} & $\begin{array}{c}\text { Após fevereiro } \\
\text { de 2013 }\end{array}$ & $\mathbf{2 0 1 4}$ & $\mathbf{2 0 1 5}$ & $\mathbf{2 0 1 6}$ & $\begin{array}{c}\text { Janeiro e } \\
\text { fevereiro de 2017 }\end{array}$ & Total \\
\hline Em andamento & 27 & 118 & $\begin{array}{c}127 \\
(9 \%)\end{array}$ & $\begin{array}{c}213 \\
(24 \%)\end{array}$ & $\begin{array}{c}92 \\
(30 \%)\end{array}$ & $\begin{array}{c}577 \\
(51 \%)\end{array}$ \\
\hline Concluído: para & 268 & 357 & 267 & 108 & 4 & 1.004 \\
devolução & $(87 \%)$ & $(72 \%)$ & $(62 \%)$ & $(22 \%)$ & $(2 \%)^{*}$ & $(53 \%)$ \\
\hline Concluído: liberado & 13 & 18 & 36 & 160 & 83 & 310 \\
$(4 \%)$ & $(4 \%)$ & $(8 \%)$ & $(33 \%)$ & $(46 \%)$ & $(16 \%)$ \\
\hline Total & $\mathbf{3 0 8}$ & $\mathbf{4 9 3}$ & $\mathbf{4 3 0}$ & $\mathbf{4 8 1}$ & $\mathbf{1 7 9}$ & $\mathbf{1 . 8 9 1}$ \\
\hline
\end{tabular}

Fonte: Elaboração própria com os dados da CAME/SES/RS.

* Apenas janeiro de 2017.

A análise do quantitativo financeiro solicitado, em processos concluídos para devolução ao Fundo Estadual de Saúde/SES/RS, por ano, das auditorias de OPM e CMS foi realizada por meio da agregação das informações, conforme se pode observar na Figura 1. 
Figura 1. Valor financeiro solicitado para devolução ao Fundo Estadual de Saúde/SES/RS, por ano de pagamento, das auditorias de órteses, próteses e materiais especiais, cirurgias múltiplas e sequenciais, no Rio Grande do Sul, de março de 2013 a janeiro de 2017

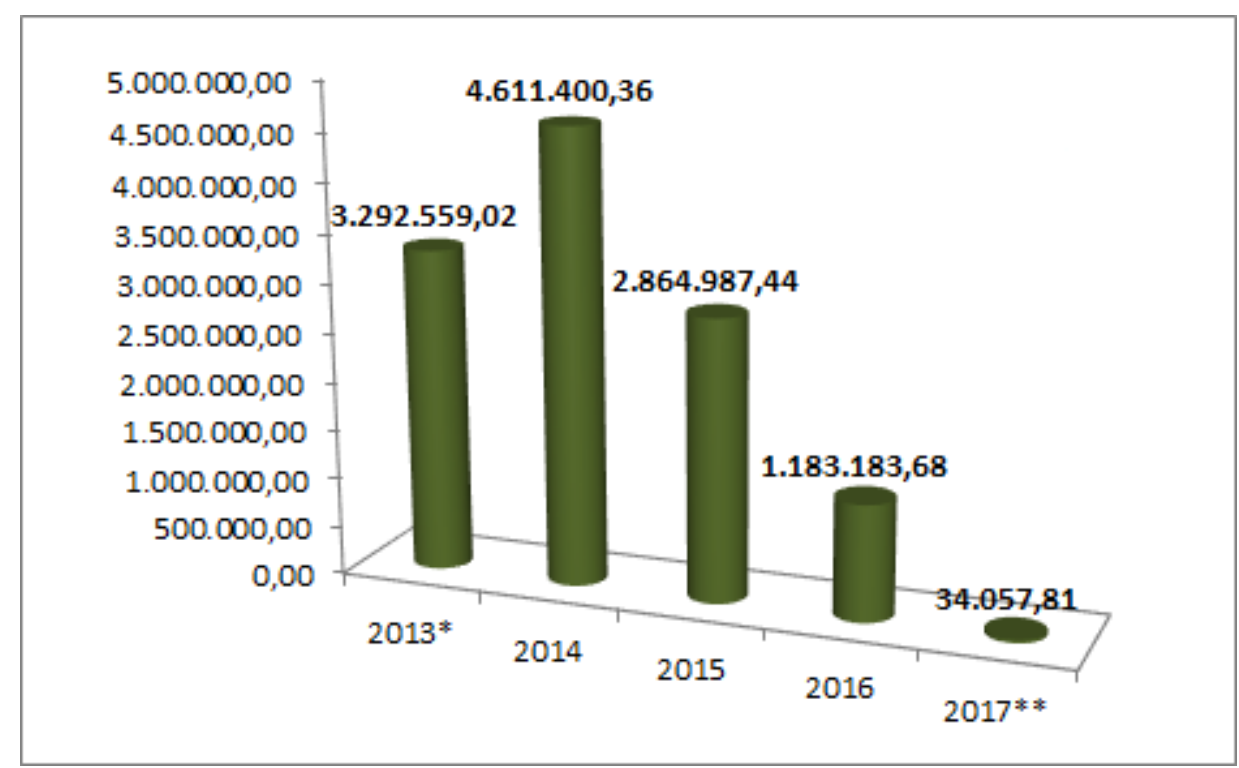

Fonte: elaboração própria com base nos dados da CAME/SES/RS.

* Dados de 2013 considerados a partir de março do respectivo ano.

${ }^{* *}$ Dados de 2017 somente o mês de janeiro do respectivo ano.

O valor financeiro referente aos processos administrativos com ordem de recolhimento (solicitação de devoluções) das auditorias de OPM e CMS não teve uma tendência clara na série histórica analisada, resultado influenciado pela presença de processos administrativos não conclusos, em especial a partir de 2014. Esse valor foi maior em 2014, decrescendo em 2015 e diminuiu ainda mais nos anos 2016 e 2017; mas tal resultado tem associação com o fato de ainda haver processos administrativos ou judiciais em andamento, conforme mencionado anteriormente.

Em sequência, foi realizada a análise por região de saúde, considerando-se para isso a população, o número de processos administrativos com ordem de recolhimento (OR), o número de instituições auditadas com OR, o valor total dos processos administrativos com OR de autorizações de internação hospitalar de OPM e CMS pagas, conforme se pode observar no Quadro 2. 
Quadro 2. População por Região de Saúde, número de processos administrativos com ordem de recolhimento $(\mathrm{OR})$, número de instituições auditadas com OR, valor total dos processos administrativos com OR de autorizações de internação hospilar de órteses, próteses e materiais especiais, cirurgias múltiplas e sequenciais pagas, no Rio Grande do Sul, de março de 2013 a janeiro de 2017

\begin{tabular}{|c|c|c|c|c|}
\hline Região de Saúde (RS) & População* & $\begin{array}{c}\text { Número de } \\
\text { processos } \\
\text { com OR }\end{array}$ & $\begin{array}{c}\text { Número de } \\
\text { instituições } \\
\text { auditadas com OR }\end{array}$ & $\begin{array}{c}\text { Valor total }(\mathrm{R} \$) \\
\text { dos processos } \\
\text { com OR }\end{array}$ \\
\hline R1 Verdes Campos & 435.021 & 40 & 6 & $212.597,36$ \\
\hline R2 Entre-Rios & 127.574 & 7 & 2 & $26.836,84$ \\
\hline R3 Fronteira Oeste & 476.748 & 159 & 7 & $1.488 .956,83$ \\
\hline R4 Belas Praias & 148.751 & 1 & 1 & $3.761,29$ \\
\hline R5 Bons Ventos & 218.625 & 14 & 2 & $121.317,59$ \\
\hline $\begin{array}{l}\text { R6 Vale do Paranhana/ Costa } \\
\text { da Serra }\end{array}$ & 225.321 & 68 & 4 & $1.520 .600,18$ \\
\hline R7 Vale dos Sinos & 799.530 & 83 & 5 & $1.372 .381,50$ \\
\hline R8 Vale Caí/ Metropolitana & 756.735 & 72 & 4 & $1.789 .059,98$ \\
\hline R9 Carbonífera/Costa Doce & 403.907 & 69 & 2 & $651.540,86$ \\
\hline R10 Capital/Vale Gravataí & 2.332 .576 & 28 & 1 & $234.379,69$ \\
\hline R11 Sete Povos Missões & 292.789 & 28 & 2 & $438.290,68$ \\
\hline R12 Portal das Missões & 156.494 & 33 & 3 & $134.626,76$ \\
\hline R13 Região da Diversidade & 231.229 & 52 & 4 & $155.445,77$ \\
\hline R14 Fronteira Noroeste & 232.754 & 51 & 8 & $102.009,97$ \\
\hline R15 Caminho das Águas & 191.333 & 61 & 7 & $352.991,10$ \\
\hline R16 Alto Uruguai Gaúcho & 238.237 & 30 & 1 & $300.603,16$ \\
\hline R17 Região do Planalto & 403.557 & 27 & 3 & $718.469,33$ \\
\hline R18 Região das Araucárias & 133.367 & 1 & 1 & 973,65 \\
\hline R19 Região do Botucaraí & 119.545 & 4 & 2 & $3.883,63$ \\
\hline R20 Rota da Produção & 166.764 & 21 & 4 & $44.146,25$ \\
\hline R21 Região Sul & 879.864 & 49 & 4 & $1.060 .737,64$ \\
\hline R22 Região Pampa & 189.638 & 43 & 1 & $902.077,84$ \\
\hline R23 Caxias e Hortênsias & 574.115 & 0 & 0 & 0,00 \\
\hline
\end{tabular}




\begin{tabular}{|l|c|c|c|r|}
\hline \multicolumn{1}{|c|}{ Região de Saúde (RS) } & População* & $\begin{array}{c}\text { Número de } \\
\text { processos } \\
\text { com OR }\end{array}$ & $\begin{array}{r}\text { Número de } \\
\text { instituições } \\
\text { auditadas com OR }\end{array}$ & $\begin{array}{r}\text { Valor total (R\$) } \\
\text { dos processos } \\
\text { com OR }\end{array}$ \\
\hline R24 Campos de Cima Serra & 99.168 & 0 & 0 & 0,00 \\
\hline R25 Vinhedos e Basalto & 298.581 & 0 & 0 & 0,00 \\
\hline R26 Uva Vale & 180.346 & 0 & 0 & $30.459,48$ \\
\hline R27 Jacuí Centro & 206.937 & 5 & 0 & 0,00 \\
\hline R28 Vale do Rio Pardo & 343.858 & 0 & 3 & $52.759,07$ \\
\hline R29 Vales e Montanhas & 219.296 & 9 & 3 & $\mathbf{2 6 7 . 2 8 1 , 8 6}$ \\
\hline R30 Vale da Luz & 124.614 & 49 & $\mathbf{8 2}$ & $\mathbf{1 1 . 9 8 6 . 1 8 8 , 3 1}$ \\
\hline Rio Grande do Sul & $\mathbf{1 1 . 2 0 7 . 2 7 4}$ & $\mathbf{1 . 0 0 4}$ & 0 & 0.000 \\
\hline
\end{tabular}

Fonte: elaboração própria com base nos dados da CAME/SES/RS; IBGE/DATASUS.

*População estimada para o ano de 2014.

Pela análise do Quadro 2, percebe-se que cinco regiões de saúde acumularam mais de um milhão de reais em processos administrativos com ordem de recolhimento de OPM/CMS , são elas: R8, 1.789.059,98 reais, com 72 processos em quatro instituições hospitalares); R6, 1.520.600,18 reais, com 68 processos em quatro instituições hospitalares; R3,,1.488.956,83 reais, com 159 processos em sete instituições hospitalares; R7, 1.372.381,50 reais, com 83 processos em cinco instituições hospitalares; e R21, 1.060.737,64 reais, com 49 processos em quatro instituições hospitalares.

Outro ponto que merece destaque é que as instituições hospitalares das regiões de saúde R23, R24, R25, R26 e R28, todas localizadas na macrorregião de saúde Serra/Vales, não tiveram nenhum processo administrativo com ordem de recolhimento de OPM/CMS no período analisado.

Ao observar as regiões de saúde com uma única instituição hospitalar com processos administrativos com ordem de recolhimento, três estabelecimentos hospitalares se destacam pelos números: a região de saúde 10 , com 28 processos administrativos, no valor de $234.379,69$ reais; a região de saúde 16 , com 30 processos administrativos no valor de $300.603,16$ reais; e região de saúde 22 , com 43 processos administrativos no valor de 902.077,84 reais (Quadro 2). 
Seriam necessárias mais informações, ou até mesmo outro estudo, para entender se essas regiões de saúde com maiores solicitações de devolução são referências para o estado do Rio Grande do Sul na área de OPM e CMS, ou se apresentaram problemas como mal preenchimento das autorizações de internações hospitalares e/ou cobrança de valores indevidos relacionados aos procedimentos realizados.

Uma ilustração da relevância da judicialização, na área estudada pode ser feita com os dados dos processos concluídos para devolução. Do total de cerca de mil processos, foram judicializados 38 registros, representando apenas $3,8 \%$ do total, mas que são impactantes em termos de valor financeiro, alcançando $1.117 .891,71$ reais, o que configura $9,33 \%$ do valor dos concluídos para devolução. Além disso, observa-se que esses processos judiciais se concentraram em quatro regiões de saúde - R6, R7, R8 e R30 -, destacando-se a região de saúde R6, no Vale do Paranhana/Costa da Serra, que obteve os maiores efeitos da judicialização, com 11 registros, totalizando aproximadamente 587 mil reais e que representaram mais de $1 / 3$ do total dessa região, que foi de 1,5 milhões. A região de saúde R7, no Vale dos Sinos, com 10 registros, totalizando aproximadamente 466 mil reais e que representaram $33,93 \%$ do valor dessa região, que foi de 1,3 milhões de reais. Vale ressaltar que as regiões de saúde $R 6$ e $R 7$ são, respectivamente, a segunda e a quarta com maiores valores solicitados para devolução.

Outro ponto a destacar é que, quando se observa a distribuição espacial de leitos da rede prestadora de serviços ao SUS no Rio Grande do Sul por região de saúde (Figura 2), e se analisa a distribuição dos processos administrativos com ordem de recolhimento de $\mathrm{AlH}$ de OPM e CMS, pagas no período desse estudo (Figura 3), nota-se visualmente que há pouca relação nessa concentração. Esse fato poderia ser melhor investigado ao se observar a oferta de serviços, ou seja, a contratualização hospitalar para o atendimento das internações para a realização de órteses, próteses e materiais especiais e cirurgias múltiplas e sequenciais - dados que este estudo não conseguiu coletar e, por isso, não se propôs estudar, mas que mereceriam ser melhores explorados. 
Figura 2. Distribuição de leitos (internação e complementares) SUS no estado do Rio Grande do Sul, por região de saúde, em março de 2017

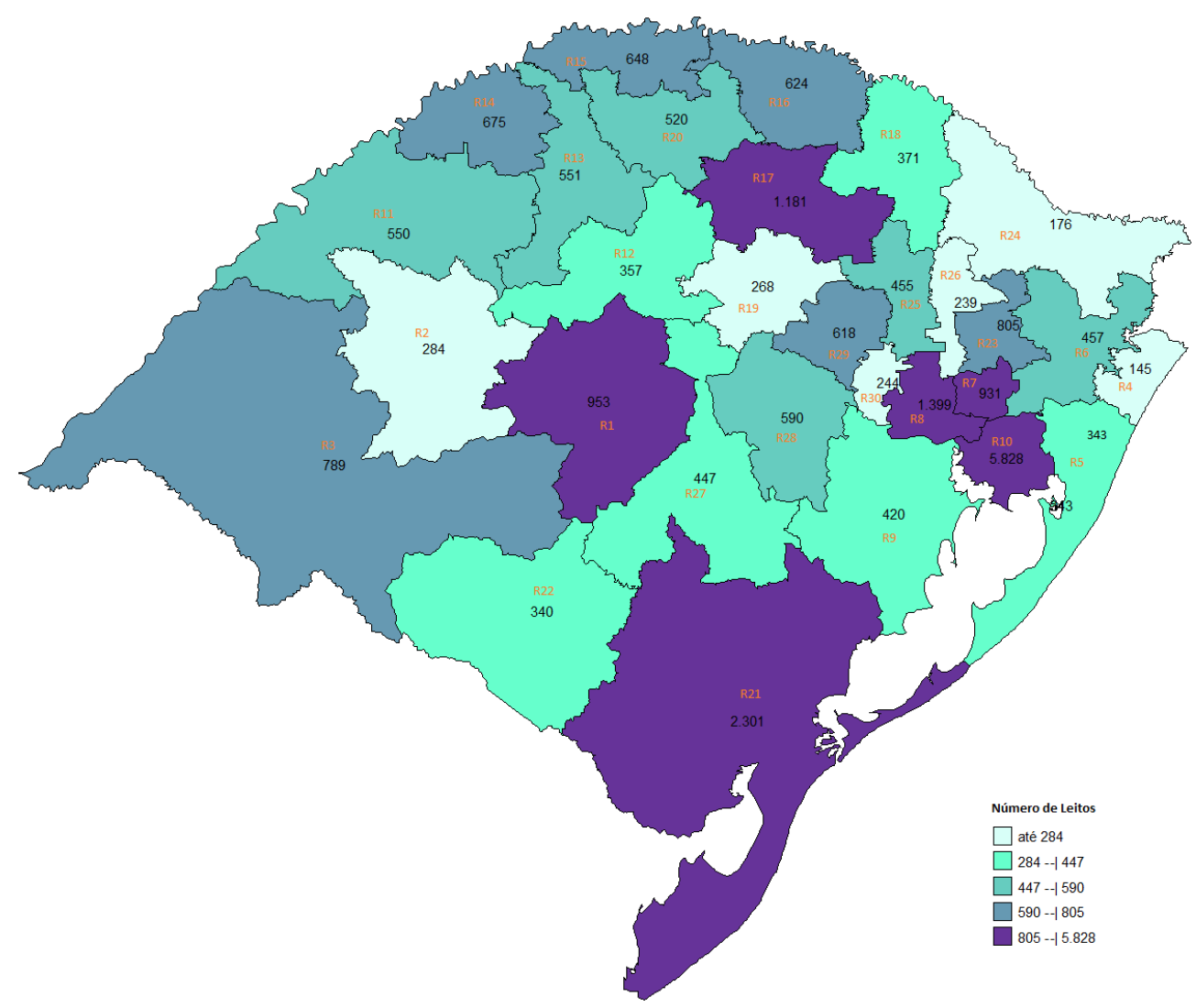

Fonte: elaboração própria com base nos dados do Cadastro Nacional de Estabelecimentos de Saúde (CNES/DATASUS). 
Figura 3. Distribuição do número de processos administrativos com ordem de recolhimento de autorização de internações hospitalares de órteses, próteses e materiais especiais, cirurgias múltiplas e sequenciais pagas, por região de saúde, no Rio Grande do Sul, de março de 2013 a janeiro de 2017

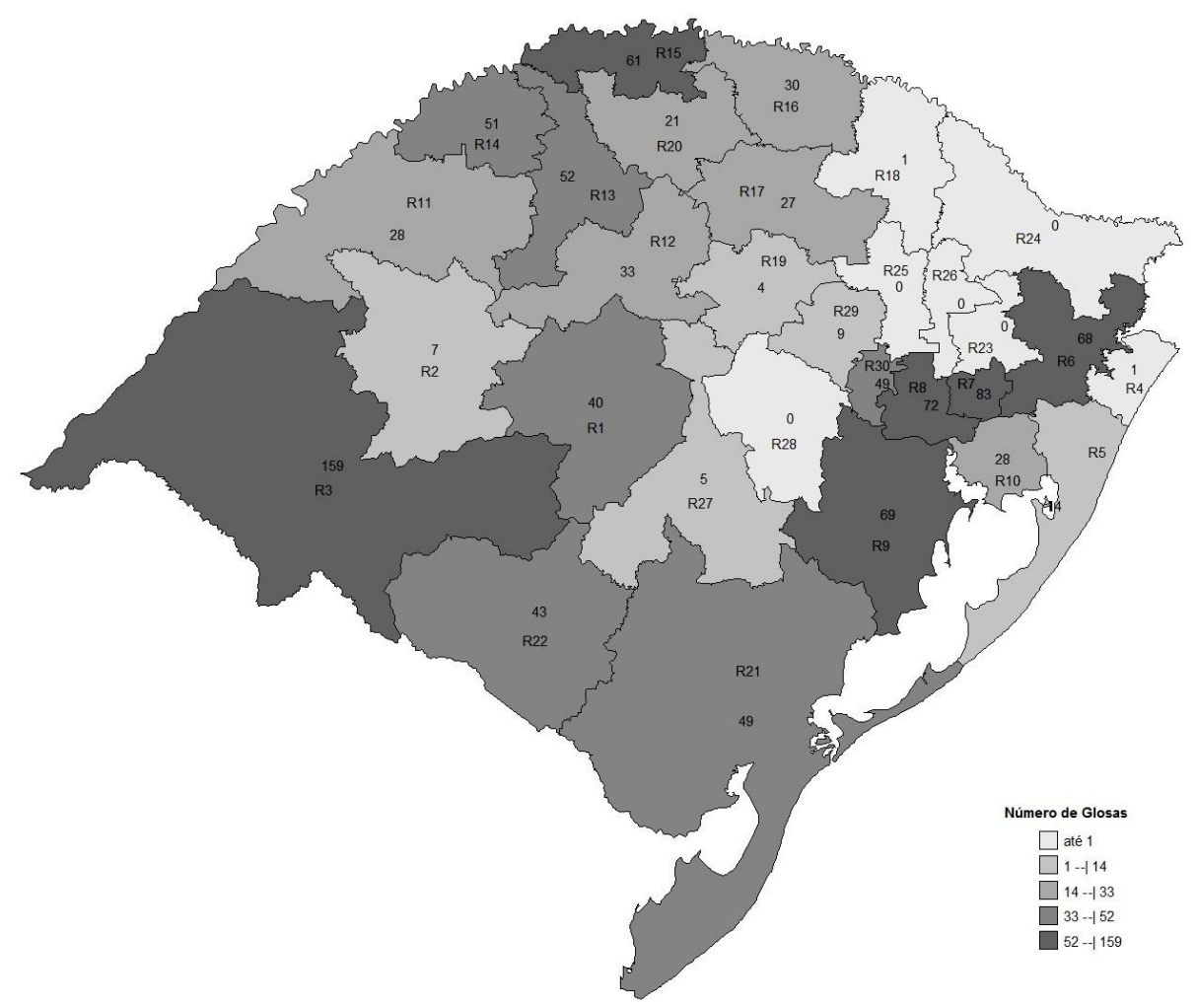

Fonte: elaboração própria com base nos dados da CAMES/SES/RS; DATASUS.

\section{Discussão}

As despesas em ações e serviços do estado do Rio Grande do Sul, na rede própria e conveniada ao Sistema Único de Saúde, no ano de 2017, foram de 3.777.766.830,37 reais (14). Mesmo considerando o valor acumulado da série histórica analisada - 11.986.188,31 reais, de processos administrativos com ordem de recolhimento, conforme o Quadro 2 - 0 valor despendido em saúde, no ano de 2017, chega a apenas $0,32 \%$.

A pertinência do trabalho advém de demonstrar a importância dos registros individuais para o monitoramento, avaliação e planejamento das ações que visem qualificar o processo de gestão, e, com isso, problematizar sobre a execução dessa atividade como uma forma de otimização dos gastos públicos em saúde por parte do Estado. 
Cabe destacar que a auditoria em saúde, no modelo executado no Brasil, parece atuar mais na lógica retrospectiva, ou seja, quase sempre é uma autopsia. $O$ ideal é que seja uma prevenção, realizada antes do problema já estar instalado.

Os valores apresentados de solicitações de devolução de recursos são relativos a processos administrativos com ordem de recolhimento de autorização de internações hospitalares de OPM e e CMS pagas. Tais valores podem já ter sido recolhidos ou permanecerem como restos a pagar pelas instituições hospitalares ao Fundo Estadual de Saúde/SES/RS, e tal fato igualmente não foi objeto do presente estudo.

Pela análise do Quadro 1, percebe-se que o percentual de processos administrativos com ordem de recolhimento, liberados após o processo de auditoria, foi inferior a $10 \%$ até o ano de 2015; no ano de 2016 subiu para mais de $30 \%$, ou seja, um aumento de situações de notificação de irregularidade. Ademais, nos anos mais recentes, os processos administrativos em andamento possuem peso maior, o que pode estar associado a aspectos da execução da auditoria ou também à judicialização do assunto pelas instituições auditadas.

Destaca-se que as atividades de auditoria em saúde analisadas têm atuado em ambiente de maior complexidade, uma vez que passaram a ser objeto de judicialização pelos prestadores auditados. Como consequência, os processos da categoria concluído para devolução foram apresentando menor peso nos anos recentes da série histórica analisada.

Marques e Almeida (15), considerando e experiência de Portugal, discutem sobre o papel do Estado na gestão da máquina pública e suas responsabilidades diante o cidadão, ao fazer a utilização dos recursos públicos de forma eficiente e eficaz. Segundo os autores, tal perspectiva passa por formas novas e efetivas de controle do gasto público e da construção, aplicação e monitoramento de estratégias que busquem atingir esse fim.

Os dados empregados nesse estudo advêm de uma planilha de registros administrativos da Coordenação Estadual de Saúde da Secretaria Estadual de Saúde do Rio Grande do Sul (SES/RS). No entanto, esses poderiam ser públicos por meio de relatórios disponíveis no Sistema de Auditoria do SUS (SISAUD/SUS), facilitando sua prestação de contas e acompanhamento pelos conselhos de saúde; esse ainda é um processo que precisa avançar.

Um estudo realizado com amostra aleatória de relatórios de auditoria de programas de saúde de 980 municípios brasileiros identificou, pela análise de regressão múltipla, que a 
experiência dos conselhos municipais de saúde estava correlacionada com reduções na incidência de corrupção nos programas de saúde pública. (16)

Entre os possíveis efeitos da auditoria, o mais corrente seria a devolução de gastos indevidos por parte do prestador de serviços, na perspectiva legal e não apenas em um sentido punitivo, uma vez que "a devolução de recursos é um ponto significativo no contexto geral de auditoria do SUS" (17).

Adicionalmente, a auditoria em saúde pode ter também um efeito educativo ou de prevenção, pois as instituições que receberam auditoria - em especial as que receberam punição - podem vir futuramente a reduzir a ocorrência do problema específico e/ou melhorar a sua gestão em relação ao cumprimento das regras. Há até mesmo um efeito de transbordamento - externalidade positiva -, em que a ação de auditoria pode vir a aprimorar o desempenho dessa atividade e da atuação do Estado no controle, monitoramento e avaliação da utilização dos recursos públicos aplicados em saúde.

Dias et al (18), ao analisar as ações da auditoria de enfermagem, utilizando a revisão sistemática da literatura, encontraram nos artigos publicados que um dos pilares que norteia as ações dessa prática é o processo educativo.

Outro estudo de revisão bibliografia, que buscou descrever a importância da auditoria como ferramenta para gestão, planejamento, monitoramento e avaliação, especificamente na atenção primária à saúde, concluiu que a auditoria pode contribuir reorientando os processos de gestão, da regulação da atenção à saúde, além de promover a equidade no acesso aos serviços e ações do Sistema Único de Saúde, com oferta de cuidados focada nas necessidades em saúde dos usuários (19).

Outro ponto a ser destacado é a qualificação das ações de auditoria para evitar processos desnecessários de judicialização em saúde. Apesar dessa via poder "ser um instrumento importante de garantia dos direitos dos cidadãos", como externalidade negativa também pode reforçar as iniquidades em saúde e provocar "dificuldades na operação cotidiana das equipes que fazem a gestão do sistema de saúde", inclusive impactando negativamente na execução orçamentária (20).

Uma questão a ser considerada como dificuldade do presente trabalho é a não disponibilização das informações sobre auditorias de OPM e CMS do estado do Rio Grande do Sul no Sistema de Auditoria do SUS (SISAUD/SUS). Cabe destacar que hoje o 
SISAUD/SUS é um sistema de informação pouco amigável, no sentido de ter muitas informações de forma não sistematizada - especificamente os relatórios - e não haver um padrão na alimentação por parte dos estados e municípios, o que dificulta a comparação e avaliação das atividades da auditoria em saúde realizadas pelos diferentes entes federativos. Talvez uma plataforma no modelo do TABNET/DATASUS poderia ajudar a minimizar essa questão.

Melo e Vaitsman (21) trazem que a qualidade dos registros sobre ações e serviços de saúde são primordiais, tanto para a avaliação quanto para a auditoria em saúde. Para as autoras, as informações podem influenciar na interpretação, nos resultados e nas conclusões dos processos de avaliação e auditoria em saúde e ambas áreas buscam dar subsídios para a melhoria da gestão do Sistema Único de Saúde.

Foram encontrados poucos estudos na literatura acadêmica que versassem sobre auditoria em saúde. Apesar de alguns trazerem a discussão da relação do tema com a melhor utilização do recurso público, nenhum demonstrou essa tese em dados quantitativos ou por região de saúde; por isso a dificuldade de comparabilidade com os dados do presente trabalho.

Outra limitação foi a falta de atualização monetária dos valores de cada autorização de internação hospitalar de OPM E CMS, uma vez que a planilha de análise dos dados só apresentava a data de registro do processo administrativo com ordem de recolhimento - já com o valor atualizado pela Taxa SELIC - e não a data real de pagamento.

Ainda cabe destacar a importância da utilização das informações em saúde para qualificar os processos de gestão e atenção, bem como o planejamento, o monitoramento e a avaliação em saúde (22). No entanto, persiste a discussão sobre a necessidade da completude das informações para a adequada utilização desses dados, uma vez que no SUS esses têm um emprego mais administrativo ou para fins de pagamento, muitas vezes não refletindo a real situação de saúde da população ou a organização dos processos de trabalho e gestão em saúde (23). 


\section{Conclusão}

O objeto deste estudo foi a análise das auditorias pós-pagamento de órteses, próteses e materiais especiais e cirurgias múltiplas e sequenciais realizadas pela Coordenação Estadual de Auditoria Médica da Secretaria Estadual de Saúde do Rio Grande do Sul.

A análise dos dados referentes às ações de auditoria em saúde nessa área ilustrou resultados que são influenciados por processos administrativos ou judiciais em andamento, bem como qualificou uma oportunidade de aprimoramento da gestão em saúde por meio da informação das regiões de saúde e instituições hospitalares que mais reincidem e que têm grandes volumes financeiros glosados.

A mudança da forma de pós-pagamento para pré-pagamento das auditorias é uma estratégia por parte do gestor público para fazer o bloqueio do pagamento, antes desse ser efetivado aos prestadores de serviço. Tal fato pode ajudar para que as ações da auditoria em saúde sejam mais eficazes e eficientes. No entanto, é preciso que existam registros específicos e individuais a fim de que as ações desenvolvidas possam ser avaliadas, qualificando assim o processo de atenção, gestão e gastos de recursos públicos no Sistema Único de Saúde.

Por fim, por este trabalho, espera-se que a discussão sobre a importância da auditoria em saúde para a gestão e para a economia da saúde possa ser aprofunda por outros estudos e pesquisadores. Dessa forma, estar-se-á buscando atingir o papel do Estado no controle da efetiva utilização dos recursos públicos e da otimização de suas ações.

\section{Referências}

1. Brasil. Ministério da Saúde, Secretaria de Gestão Estratégica e Participativa. Política Nacional de Gestão Estratégica e Participativa no SUS - ParticipaSUS. Ministério da Saúde, Secretaria de Gestão Estratégica e Participativa. 2. ed. Brasília: Editora do Ministério da Saúde; 2009. 44 p. Disponível em: http://bvsms.saude.gov.br/bvs/publicacoes/politica_estrategica_participasus_2ed.pdf. [Acesso em 29 mar. 2018].

2. Ayach C, Moimaz SAS, Garbin CAS. Auditoria no Sistema Único de Saúde: o papel do auditor no serviço odontológico. Saúde e Sociedade. 2013, 22(1): 237-248.

3. Costa AMC. A auditoria interna nos municípios portugueses. [Dissertação]. Coimbra: Mestrado em Contabilidade e Finanças, Faculdade de Economia, Universidade de Coimbra; 2008. Disponível em: https://estudogeral.sib.uc.pt/handle/10316/17846. [Acesso em 15 jun. 2018]. 
4. Brasil. Ministério da Saúde, Presidência da República. Casa Civil. Subchefia para Assuntos Jurídicos. Lei № 8.080, de 19 de setembro de 1990. Dispõe sobre as condições para a promoção, proteção e recuperação da saúde, a organização e o funcionamento dos serviços correspondentes e dá outras providências. Diário Oficial [da] República Federativa do Brasil, Poder Executivo, Brasília, DF; 20 set. 1990. Disponível em:

http://www.planalto.gov.br/ccivil_03/leis/L8080.htm. [Acesso em 08 nov. 2017].

5. Brasil. Ministério da Saúde, Secretaria de Gestão Estratégica e Participativa.

Departamento Nacional de Auditoria do SUS. Vamos conversar sobre auditoria do SUS? Ministério da Saúde, Secretaria de Gestão Estratégica e Participativa, Departamento Nacional de Auditoria do SUS. Brasília: Ministério da Saúde; 2013. 20 p. Disponível em: http://sna.saude.gov.br/download/cartilhaWEB_SUS_2111.pdf. [Acesso em 15 abr. 2018].

6. Brasil. Ministério da Saúde, Secretaria de Gestão Estratégica e Participativa.

Departamento Nacional de Auditoria do SUS. Princípios, diretrizes e regras da auditoria do SUS no âmbito do Ministério da Saúde. Ministério da Saúde, Secretaria de Gestão Estratégica e Participativa, Departamento Nacional de Auditoria do SUS. Brasília: Ministério da Saúde; 2017. 48 p. Disponível em:

http://bvsms.saude.gov.br/bvs/publicacoes/principios_diretrizes_regras_auditoria_sus.pdf. [Acesso em 15 abr. 2018].

7. Rio Grande do Sul, Lei Estadual № 11.867, de 11 de dezembro de 2002. Cria os procedimentos administrativos do Sistema Estadual de Auditoria e as medidas aplicáveis às irregularidades ocorridas no Sistema Único de Saúde do Estado do Rio Grande do Sul, e dá outras providências. Porto Alegre; 2002. Disponível:

http://www.al.rs.gov.br/filerepository/repLegis/arquivos/11.867.pdf. [Acesso em 21 abr. 2018].

8. Ferraz OLM, Vieira FS. Direito à Saúde, Recursos Escassos e Equidade: Os Riscos da Interpretação Judicial Dominante. Dados. 2009, 52(1):223-251.

9. Yin RK. Estudo de caso: planejamento e métodos. Tradução: Cristhian Matheus Herrera. 5. ed. Porto Alegre: Bookman; 2015. 290 p.

10. Brasil. Ministério da Saúde, Departamento de Informática do SUS (DATASUS). Disponível em: http://datasus.saude.gov.br/informacoes-de-saude/tabnet. [Acesso em 15 abr. 2018].

11. Rio Grande do Sul, Secretaria da Saúde. Resolução CIB/RS N 555 de 19 de setembro de 2012. Altera a configuração e a quantidade de Regiões de Saúde no Rio Grande do Sul e institui as Comissões Intergestores Regionais - CIR. Porto Alegre; 2012. Disponível em: http://www.saude.rs.gov.br/upload/arquivos/201703/28155806-resolucao-cib-555-12.pdf. [Acesso em 07 abr. 2018].

12. Rio Grande do Sul, Secretaria da Saúde. Resolução CIB/RS No 499 de 25 de setembro de 2014. Altera a configuração das Regiões de Saúde: R15 - Caminho das Águas, R20 - 
Rota da Produção e R17 - Região do Planalto. Porto Alegre; 2014. Disponível em: http://www.saude.rs.gov.br/upload/arquivos/201703/28155805-resolucao-cib-499-14.pdf. [Acesso em 07 abr. 2018].

13. Brasil. Tribunal de Contas da União, Atualização de débito. Sistema Atualização de Débito. Disponível em: https://contas.tcu.gov.br/debito/Web/Debito/CalculoDeDebito.faces. [Acesso em 18 abr. 2018].

14. Rio Grande do Sul, Secretaria da Saúde. Relatório Anual de Gestão de 2017. Porto Alegre; 2018. Disponível em:

http://www.saude.rs.gov.br/upload/arquivos/carga20180402/03170232-rag-2017-finalconsolidado.pdf. [Acesso em 21 abr. 2018].

15. Marques MCC, Almeida JJM. Auditoria no sector público: um instrumento para a melhoria da gestão pública. Revista Contabilidade \& Finanças. 2004, 15(35):84-95.

16. Avelino G, Barberia LG, Biderman C. Governance in managing public health resources in Brazilian municipalities. Health Policy and Planning. 2014, 29(6):694-702.

17. Brasil. Ministério da Saúde, Secretaria de Gestão Estratégica e Participativa.

Departamento Nacional de Auditoria do SUS. Devolução de recursos em auditoria do SUS: (orientações técnicas). Ministério da Saúde, Secretaria de Gestão Estratégica e Participativa, Departamento Nacional de Auditoria do SUS. 1. ed. atual. Brasília: Ministério da Saúde; 2014. 86 p. Disponível em:

http://sna.saude.gov.br/download/proj_devolucao\%20de\%20recurso.pdf. [Acesso em 20 abr. 2018].

18. Dias TCL, Santos JLG, Cordenuzzi OCP, Prochnow AG. Auditoria em enfermagem: revisão sistemática da literatura. Revista Brasileira de Enfermagem. 2011, 64(5):931-937.

19. Silva CA, Pedroni LCBR, Sousa MR, Marra RP, Bahia RR, Guerra VA. Auditoria como instrumento de gestão na Atenção Primária à Saúde. GERAIS: Revista de Saúde Pública do SUS/MG. 2015, 3(1):72-80.

20. Chagas CP, Santos FP. Efeitos do gasto com a judicialização da saúde no orçamento da Secretaria Estadual de Saúde do Distrito Federal entre 2013 e 2107. Revista Cadernos Ibero-Americanos de Direito Sanitário. 2018, 7(2):147-172.

21. Melo MB, Vaitsman J. Auditoria e avaliação no sistema único de saúde. São Paulo em Perspectiva. 2008, 22(1):152-164.

22. Chaves LDP, Ferreira JBB, Camelo SHH, Balderrama P, Tanaka OY. Reflexões acerca de Sistemas de Informação em Saúde, Pesquisa Avaliativa e Enfermagem. Enfermaria Global. 2014, 13(34):303-312. 
Cadernos Ibero-Americanos

de Direito Sanitário

Cuadernos lberomericanos

de Derecho Sanitario

23. Santos SR, Ferreira JA, Santa Cruz EMM, Leite EMAM, Pessoa JCS. Sistema de informação em saúde: gestão e assistência no Sistema Único de Saúde. Cogitare Enfermagem. 2014, 19(4):833-840.

Como citar esse artigo:

Quevedo ALA, Leal RM. Auditoria em saúde e economia da saúde: análise de um estudo de caso. Cadernos IberoAmericanos de Direito Sanitário. 2019 abr./jun.; 8(2): 44-63. 